\title{
Inferring Driver's Turning Direction through Detection of Error Related Brain Activity
}

\author{
Huaijian Zhang, Ricardo Chavarriaga, Lucian Gheorghe, and José del R. Millán
}

\begin{abstract}
This work presents EEG-based Brain-computer interface (BCI) that uses error related brain activity to improve the prediction of driver's intended turning direction. In experiments while subjects drive in a realistic car simulator, we show a directional cue before reaching intersection, and analyze error related EEG potential to infer if the presented direction coincides with the driver's intention. In this protocol, the directional cue provides an initial estimation of the driving direction (based on EEG, environmental or previous driving habits), and we focus on the recognition of error-potentials it may elicit. Experiments with 7 healthy human subjects yield an average classification $0.69 \pm 0.16$, which confirms the feasibility of decoding these signals to help estimating driver's turning direction. This study can be further exploited by intelligent cars to tune their driving assistant systems to improve their performance and enhance the driving experience.
\end{abstract}

\section{INTRODUCTION}

Automobile driving is a complex task that requires a high level of attention. Around $10 \%$ to $33 \%$ traffic accidents are due to human factors, e.g. conversation, wireless communication or music [1]. So far, driving assistant systems have been developed to avoid potential traffic accidents or reduce driving stress by monitoring driving actions (e.g. steering, braking and accelerating), environmental conditions (e.g. car's location or distance from other automobiles), and driver's physiological signals (e.g. EEG, ECG and EOG) [2] [3]. Combination of these information can be beneficial to improve driving experience.

Brain-computer interfaces (BCI) decode brain signals to monitor people's cognitive states or predict actions. Alternatively, BCI systems can be used to estimate driver's action intention or cognitive states, and provide this information to a driving assistant system in order to increase safety or reduce driving complexity. For example, BCI could be used to detect whether the driver is paying attention on driving, or predict his/her intended action in specific situations (e.g. in front of an intersection, facing traffic lights and lane changing). The present study aims to use error related brain activity before reaching interaction to infer the driver's intended turning direction.

Error processing is a basic function of the human brain, which plays a key role in integrating different cognitive processes and adjusting performance [4]. Multiple studies have

\footnotetext{
*This study was supported by Nissan Motor Co. Ltd., and carried out under the "Research on Brain Machine Interface for Drivers" project.

H. Zhang, R. Chavarriaga, L. Gheorghe, and J. d. R. Millán are with the Defitech Foundation Chair in Non-Invasive Brain-Machine Interface, Center for Neuroprosthetics, École Polytechnique Fédérale de Lausanne (EPFL), Switzerland. L. Gheorghe is also with Mobility Services Laboratory, Nissan Research Center, Nissan Motor Co., Japan. huaijian.zhang@epfl.ch jose.millandepfl.ch
}

reported event related potentials (ERP) when people execute error responses, which distributes in frontocentral and begins in adolescence and increases in early adulthood [5], reflecting coordinated theta band activities [6] [7]. Neuroimaging studies localized this activity in medial frontal cortex (MFC), particularly anterior cingulate cortex [5]. The amplitude of the ERP is related to the probability of the unexpected event, where the lower expectation of error event generates stronger amplitude [8] [9]. Error related brain activity has also been verified when people observe error events, which is about 200 to $300 \mathrm{~ms}$ after occurrence of unexpected events, and might have similar neural mechanisms as response error [10] [11]. BCI researchers have used single trial error related brain activity to improve the interaction quality between human brain and external devices [9] [12] [13].

In this work, we apply single trial error detection in driving task, for the purpose of improving estimation of driver's turning direction. We record EEG signals while subjects are driving a car simulator. Before reaching road intersections, a driving assistant system presented a directional cue indicating a possible direction to turn. We analyze the elicited signals both in the temporal and spectral domain in order to find possible difference between error and correct conditions. Furthermore, we assess single trial classification of error/correct related potentials, in order to testing the feasibility of exploiting these signals in a driving scenario.

\section{METHODS}

\section{A. Experimental protocols}

Seven subjects (six male, with age $27.1 \pm 3.2$ ) participated in the experiment. All subjects had normal or corrected-tonormal vision. No known neurological or psychiatric diseases were reported among them. Six subjects had driving licenses and four of them were active drivers. As shown in Figure 1.A, the subject sat in a car simulator in front of a projector screen, with size $1.6 \mathrm{~m}$ (width) $\times 1.25 \mathrm{~m}$ (height). The car simulator included steering, braking pedal and accelerating pedal to change direction, stop and speed up during driving. The distance between the subject and the screen was $1.8 \mathrm{~m}$. We designed a virtual environment of a small town with frequent intersections for the experiment. There was no other automobile in the environment. During the experiment, the subject was requested to fixate eyes on the center of the screen, and drive the car following street boards located on top of intersections, as shown in Figure 1.B. Once the car was about $80 \mathrm{~m}$ from an intersection, a warning cue composed of three gray arrows (pointing left, up and right) appeared at the lower part of the wind shield. One second later, one 

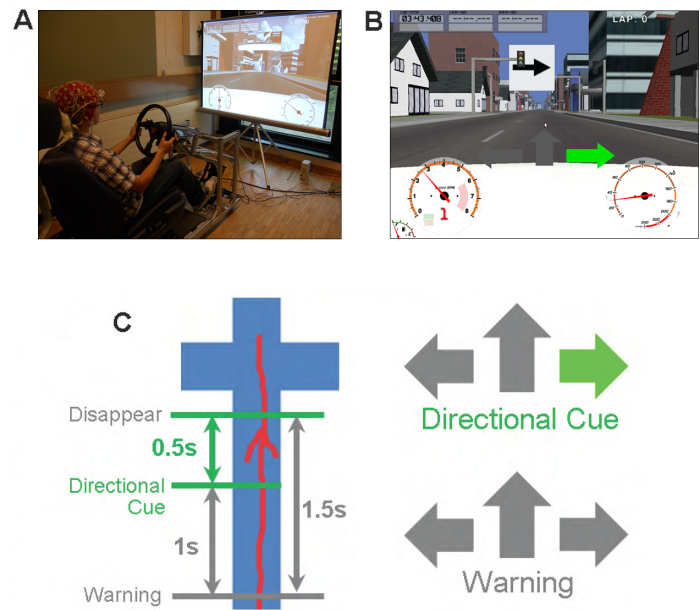

Fig. 1. Experimental protocol. A. Subject sat in car simulator and drove in virtual reality environment with EEG recording. B. Screenshot of driving environment. When the car was in front of an intersection, a directional cue appeared to evoke correct/error related potential. It was a correct trial in this case. White board indicated the real driving direction. C. Timeline of visual warning and directional cue. Visual warning (three gray arrows) appeared to indicate a new trial $80 \mathrm{~m}$ before the intersection. Directional cue appeared (green arrow) $1 \mathrm{~s}$ later to guess driver's turning direction. After $0.5 \mathrm{~s}$ of directional cue, all visual arrows disappeared.

of the arrows was highlighted in green, indicating one of the possible driving direction (directional cue). All visual cues disappeared $500 \mathrm{~ms}$ after directional cue, as shown in Figure 1.C. The probability of the green arrow to be the same as the street board was $70 \%$ to simulate the estimation of directional intention. We limited the driving speed to $60 \mathrm{~km} / \mathrm{h}$ in the driving program. Each session consisted of 30 trials (i.e. intersections) and lasted about 9-12 min, depending on the driving speed. Each subject performed 5 sessions, yielding 150 trials in total. Further study would combine the error detection system with other direction estimation approaches, which could be based on environment, driving habits or EEG signals. Here, we assume those systems could perform at an estimation accuracy of $70 \%$.

\section{B. Signal Acquisition}

EEG signals were recorded from 64 channels during driving, according to the extended 10/20 system using a Biosemi Active Two system. The sampling rate of the EEG recording was $2048 \mathrm{~Hz}$. We downsampled the signal to $256 \mathrm{~Hz}$ for further analysis. Driving parameters (angle of brake pedal, accelerate pedal and steer) were recorded with a sampling rate of $256 \mathrm{~Hz}$ and synchronized with EEG data by parallel port trigger for each trial.

\section{Signal Preprocessing and Statistical Analysis}

We did an offline analysis in this study. Multi-channel EEG signals were filtered using 4th order Butterworth filter between 1 and $10 \mathrm{~Hz}$ [9]. We used common average reference (CAR) to remove background brain activity across all recorded channels. 41 channels were considered for further analysis, since peripheral channels were removed to avoid any movement artifacts. We extracted correct and error trials according to the onset of directional cue, which was considered as the origin $(t=0 \mathrm{~s})$ of the trials. Single trials within $2.2 \mathrm{~s}$ were extracted from the preprocessed EEG data, which were between $-1.2 \mathrm{~s}$ and $1 \mathrm{~s}$. After extracting trials from all subjects, we tested the significance of difference between correct and error conditions by two-sample t-test with significance level $5 \%$. The test was performed across time to find out the temporal dynamics of the significance of ERP. Statistical test of two-sample t-test was also applied at the subject level. We did this test in temporal domain, checking whether there was significant difference between correct and error at a specific time point, so we did not do correction of the test.

Power spectral density (PSD) of selected EEG electrode was computed across time by a sliding window, in order to inspect the dynamics of frequency components. The size of the sliding window was $400 \mathrm{~ms}$, and the overlapping of the sliding window was $95 \%$ to obtain smooth results. We used Yule-Walker equation to solve the auto-regressive coefficients within a sliding window to obtain PSD. Statistical test (paired Wilcoxon signed rank test with significance level 5\%) was computed in time-frequency domain for all the pixels (as shown in 2.C) to specify the location (time and frequency) of significant difference between two conditions across all subjects. Non-parametric method (Wilcoxon signed rank test) was applied, since there was only 7 samples (subjects) in each group.

\section{Classification}

Classification between correct and error was performed based on the ERP waveforms. ERPs between $0.2 \mathrm{~s}$ to $0.7 \mathrm{~s}$ from $41 \mathrm{EEG}$ electrodes (exclude peripheral electrodes) were chosen for classification. We specified the time range from $0.2 \mathrm{~s}$ to $0.7 \mathrm{~s}$, since almost all discriminant information between correct and error was located in this range (see results of statistical tests). The 50 most discriminant features were selected for classification using canonical variate analysis, which is a supervised filter method for feature selection [14].

Linear discriminant analysis (LDA) was applied to classify correct and error trials for each subject. We used 10-fold cross validation to evaluate classification performance. Since the trial numbers for the two classes were not balanced, i.e. only $30 \%$ of them were error trials, we would show the results in receiver operating characteristic (ROC) space, quantified by area under curve (AUC).

\section{RESULTS}

\section{A. ERP and Spectral Analysis}

Grand average of ERP across all seven subjects are shown in Figure 2.A. EEG trials are segmented from $1.2 \mathrm{~s}$ before to $1 \mathrm{~s}$ after the directional cue, including the visual warning at $-1 \mathrm{~s}$. From the figure, we can observe that both correct and error trials exhibit a strong evoked potential around $250 \mathrm{~ms}$ after presenting the first visual warning (three gray arrows). As expected, the waveforms of correct and error 
A

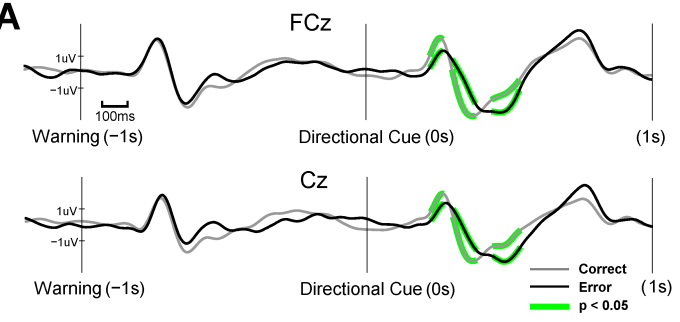

B
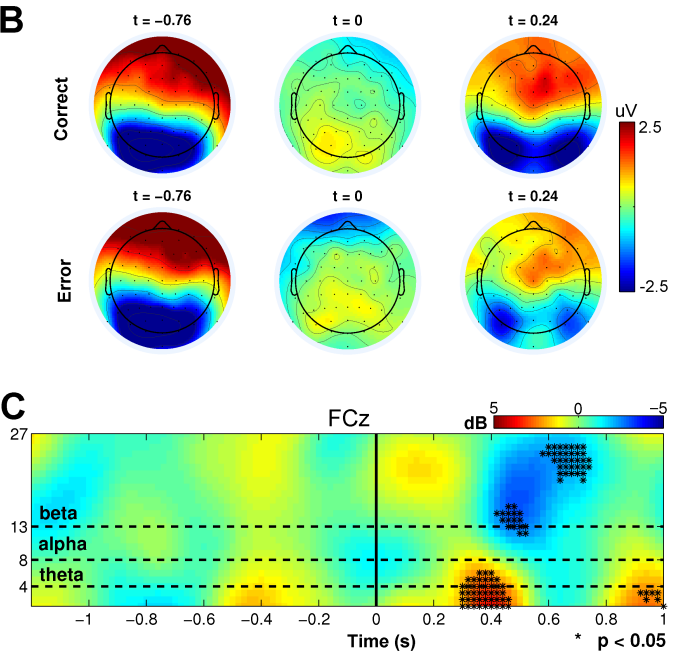

Fig. 2. A. Grand average of ERP for both correct and error conditions at electrodes $\mathrm{FCz}$ and $\mathrm{Cz}$. EEG signals are filtered between 1 to $10 \mathrm{~Hz}$. Gray curves represent correct condition, dark curves represent error condition, and green thick curves indicate significant difference $(\mathrm{p}<0.05)$ between two conditions (two-sample t-test). B. Spatial topoplots for two conditions at time points $-0.76 \mathrm{~s}, 0 \mathrm{~s}$ and $0.24 \mathrm{~s}$. C. Difference of power spectral density between error and correct at electrode $\mathrm{FCz}$ (error - correct). Asterisks indicate $\mathrm{p}<0.05$ by paired Wilcoxon signed rank across subjects.

conditions are very similar, and no significant difference cn be found ( $p>0.05$, two sample t-test). After the directional cue (i.e. onset of the green arrow), there is a positive peak around $250 \mathrm{~ms}$ followed by a negative peak for both correct and error conditions. The positive peak is higher in correct condition. About $500 \mathrm{~ms}$ after the directional cue, there is a second positive inflection peaking around $700 \mathrm{~ms}$. Significant differences $(\mathrm{p}<0.05$, two sample $\mathrm{t}$-test $)$ are found between $200 \mathrm{~ms}$ and $600 \mathrm{~ms}$ in medial central electrodes electrodes, e.g. $\mathrm{FCz}$ and $\mathrm{Cz}$ in Figure 2.A, which are denoted by the thick green line. Statistical significances $(\mathrm{p}<0.05$, two sample t-test) can also be found from six out of all seven subjects. This figure illustrates two channels as examples here. Significant differences are also found in other brain sites.

The topographic representation of the brain activity for both correct and error conditions are shown in Figure 2.B. Selected time points $(-0.74 \mathrm{~s}$ and $0.24 \mathrm{~s})$ correspond to the positive peaks of the ERPs after both warning and directional cue. We show them here to compare the responses between warning and directional cues. Both conditions present high activity in prefrontal regions and decreased activity in parietal and occipital sites after visual warning $(\mathrm{t}=-0.76 \mathrm{~s})$.
Medial frontal activity is strengthened and posterior lateral parietal is depressed after the directional cue $(\mathrm{t}=0.24 \mathrm{~s})$ for both conditions. The error condition presents lower amplitude than the correct condition. The spatial distribution of brain activity after the directional cue is located more caudal than that after the warning cue, particularly in the error condition.

The difference of PSD between the two conditions (error - correct) at electrode $\mathrm{FCz}$ is illustrated in Figure 2.C. Higher delta and theta power could be found in the error condition starting from $250 \mathrm{~ms}$ after directional cue, which is statistical significant across subjects $(\mathrm{p}<0.05$, paired Wilcoxon signed rank test). In addition, depression of the beta power in the error condition can be seen about $0.4 \mathrm{~s}$ after the directional cue, which is also significant $(\mathrm{p}<0.05$, paired Wilcoxon signed rank test). Modulation of theta and beta bands are consistent with previous studies of error related brain activity [15] [16].

\section{B. Classification}

Results of classification (ROC curves) are shown in Figure 3. In the figure, $x$ axis indicates the false positive rate (i.e. misclassified error trials), whereas $y$ axis denotes the true positive rate (i.e. properly classified correct trials). Perfect classification corresponds to an AUC equal to 1, and 0.5 means completely random performance. Using the 50 most informative features from 41 channels between $0.2 \mathrm{~s}$ and $0.7 \mathrm{~s}$, the average AUC across all subjects is 0.690 (LDA classifier with 10 -fold cross validation). All subjects exhibit results above 0.5, where the lowest AUC is 0.531. Large variability can be observed across subjects: Two subjects exhibit high performance (AUC $=0.925$ and 0.845 ), while three of them have low performance, with AUC equal to $0.531,0.543$ and 0.571 . Four out of seven subjects (4/7) have significantly higher AUC than random. In general, the classification accuracy for error trials is relatively lower than correct trials (averaged accuracy equal to 0.591 and 0.727 for error and correct trials, respectively). It should be noticed that given the experimental protocol there are more correct trials (around 70\%).

\section{DISCUSSIONS AND CONCLUSIONS}

Single trial detection of error related brain activity is usually combined with other systems to improve the performance of brain state recognition. Previous studies adopted error detection in a BCI system to improve its performance [13]. Our study suggests that it is feasible to improve the estimation of driver's turning direction by detecting error related brain activity. In the future, this information can be combined with other systems as an improvement of in-car action detection. In the current study, most subjects obtain classification accuracies higher than random, indicating that the single trial detection can help to improve the brain state recognition during driving.

Error related activity is observed in this study around $250 \mathrm{~ms}$ after the stimulus, which is consistent with previous 


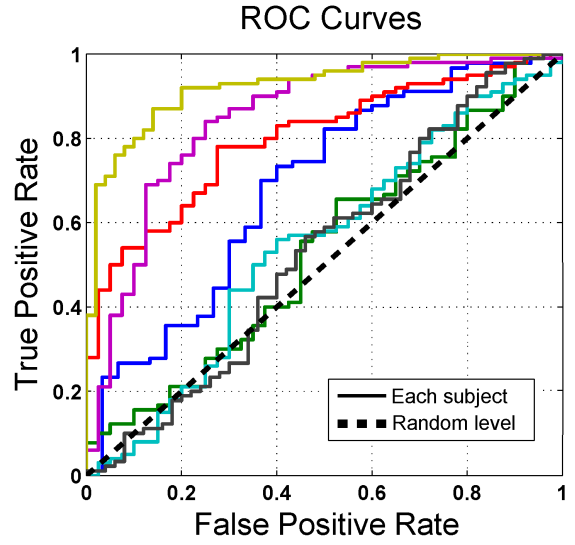

Fig. 3. Classification results (ROC curves) between error and correct trials for all seven subjects. Each line represents one subject. Random level corresponds to the diagonal.

findings [10] [11]. Our results also indicate that error condition elicits higher activity in theta rhythm and lower activity in beta rhythm, which reflects that oscillatory powers are modulated during brain error processing [15] [16].

In this study, we chose the ERP from 0.2-0.7 $\mathrm{s}$ as the input of classification because they are related to the differences between error and correct condition, as demonstrated in Figure2.A. Although the obtained accuracy is above random for most subjects, it may still be low for practical applications. The variability of the classification performances across subjects may be caused by the varying driving experience. Subjects who had participated in the experiment before could generate higher accuracy. We compared current results with previous study of error recognition with a moving cursor protocol, whose classification accuracies were $75.81 \%$ and $63.21 \%$ for correct and error trials when the error rate was 0.2 , and the accuracies were $64.42 \%$ and $59.36 \%$ for correct and error when the error rate was 0.4 [9]. This study obtain accuracies $72.7 \%$ and $59.1 \%$ with error rate 0.3 , indicating that error recognition in driving task is feasible and comparable with simple error monitoring task.

To further our study, we will explore other features for the classification. Spectral information might be helpful for us to improve the discrimination performance, since we have found statistical significance between two conditions. Moreover, causal influences between EEG electrodes, which can be obtained by multivariate auto-regressive models, can also be used as features for the discrimination, which seems to provide extra information for the classification task [17].

So far we used visual cues to evoke error related potential, which may increase the visual burden during driving. We will study other feedback modalities, such as auditory or tactile, which have been reported as stimuli to evoke brain error processing [12] [18]. Additionally, feature extraction and classification in the present study are applied offline. Future work will also address online implementation of error/correct classification in driving task, as well as real car experiments.

\section{ACKNOWLEDGEMENTS}

We would like to thank all subjects that have participated in the experiments.

\section{REFERENCES}

[1] T. A. Ranney, "Driver distraction: A review of the current state-ofknowledge," in US Department of Transportation National Highway Traffic Safety Administration, 2008.

[2] C. H. Chuang, P. C. Lai, L. W. Ko, B. C. Kuo, and C.-T. Lin, "Driver's cognitive state classification toward brain computer interface via using a generalized and supervised technology," in The 2010 International Joint Conference on Neural Networks, Jul. 2010, pp. 1-7.

[3] S. Haufe, M. S. Treder, M. F. Gugler, M. Sagebaum, G. Curio, and B. Blankertz, "EEG potentials predict upcoming emergency brakings during simulated driving," Journal of Neural Engineering, vol. 8, no. 5, pp. 1-11, Oct. 2011.

[4] C. B. Holroyd and M. G. Coles, "The neural basis of human error processing: reinforcement learning, dopamine, and the error-related negativity," Psychology Review, vol. 109, no. 4, pp. 679-709, 2002.

[5] S. F. Taylor, E. R. Stern, and W. J. Gehring, "Neural systems for error monitoring: Recent findings and theoretical perspectives." Neuroscientist, vol. 13, no. 2, pp. 160-172, Apr. 2007.

[6] P. Luu, T. Flaisch, and D. M. Tucker, "Medial frontal cortex in action monitoring." The Journal of Neuroscience, vol. 20, no. 1, pp. 464-469, Jan. 2000.

[7] L. T. Trujillo and J. J. B. Allen, "Theta EEG dynamics of the errorrelated negativity." Clinical Neurophysiology, vol. 118, no. 3, pp. 645668, Mar. 2007.

[8] M. Falkenstein, J. Hoormann, S. Christ, and J. Hohnsbein, "ERP components on reaction errors and their functional significance: a tutorial." Biological Psychology, vol. 51, no. 2-3, pp. 87-107, 2000.

[9] R. Chavarriaga and J. d. R. Millán, "Learning from EEG error-related potentials in noninvasive brain-computer interfaces," Transactions on Neural Systems and Rehabilitation Engineering, vol. 18, no. 4, pp. 381-388, 2010

[10] W. H. R. Milner, J. Brauer, H. Hecht, R. Trippe, and M. G. H. Coles, "Parallel brain activity for self-generated and observed errors." Errors, Conflicts, and the Brain. Current Opinions on Performance Monitoring (eds. Ullsperger, M. \& Falkenstein, M.), pp. 124-129, 2004.

[11] H. T. van Schie, R. B. Mars, M. G. H. Coles, and H. Bekkering, "Modulation of activity in medial frontal and motor cortices during error observation." Nature Neuroscience, vol. 7, no. 5, pp. 549-554, May 2004.

[12] X. Perrin, R. Chavarriaga, C. Ray, R. Siegwart, and J. d. R. Millán, "A comparative psychophysical and EEG study of different feedback modalities for human-robot interaction," in ACM/IEEE Conf on Human-Robot Interaction HRIO8, Amsterdam, Netherlands, 2008.

[13] P. W. Ferrez and J. d. R. Millán, "Error-related EEG potentials generated during simulated brain-computer interaction," IEEE Transactions on Biomedical Engineering, vol. 55, no. 3, pp. 923-929, 2008.

[14] F. Galán, P. W. Ferrez, F. Oliva, J. Guàrdia, and J. d. R. Millán, "Feature extraction for multi-class BCI using canonical variates analysis," in IEEE Int Symp Intelligent Signal Processing, 2007.

[15] M. X. Cohen, K. R. Ridderinkhof, S. Haupt, C. E. Elger, and J. Fell, "Medial frontal cortex and response conflict: evidence from human intracranial EEG and medial frontal cortex lesion." Brain Research, vol. 1238, pp. 127-142, Oct 2008.

[16] T. Koelewijn, H. T. van Schie, H. Bekkering, R. Oostenveld, and O. Jensen, "Motor-cortical beta oscillations are modulated by correctness of observed action." Neuroimage, vol. 40, no. 2, pp. 767-775, Apr. 2008.

[17] H. Zhang, R. Chavarriaga, L. A. Gheorghe, and J. d. R. Millán, "Improved recognition of error related potentials through the use of brain connectivity features." in 34th Annual International Conference of the IEEE Engineering in Medicine and Biology Society (EMBC'12), 2012.

[18] R. Chavarriaga, X. Perrin, R. Siegwart, and J. d. R. Millán, "Anticipation- and Error-related EEG Signals during Realistic HumanMachine interaction: A Study on Visual and Tactile Feedback," in 34th Annual International Conference of the IEEE Engineering in Medicine and Biology Society (EMBC'12), 2012. 